\title{
A New Approach for Studying Bond Rupture/Closure of a Spiro Benzopyran Photochromic Material: Reactivity Descriptors Derived from Frontier Orbitals and DFT Computed Electrostatic Potential Energy Surface Maps
}

\author{
M. S. A. Abdel-Mottaleb and Sarah N. Ali \\ Nano-Photochemistry and Solarchemistry Labs, Department of Chemistry, Faculty of Science, Ain Shams University, \\ Abbassia, Cairo 11566, Egypt \\ Correspondence should be addressed to M. S. A. Abdel-Mottaleb; phochem08@photoenergy.org
}

Received 1 November 2015; Revised 27 January 2016; Accepted 11 February 2016

Academic Editor: Mark van Der Auweraer

Copyright (C) 2016 M. S. A. Abdel-Mottaleb and S. N. Ali. This is an open access article distributed under the Creative Commons Attribution License, which permits unrestricted use, distribution, and reproduction in any medium, provided the original work is properly cited.

\begin{abstract}
This paper focuses on computations technique within the framework of the TD-DFT theory for studying the relationship between structure-properties of reversible conversion of photochromic materials. Specifically, we report on $1^{\prime}, 3^{\prime}$-dihydro- 8 -methoxy-1 $1^{\prime}, 3^{\prime}, 3^{\prime}$ trimethyl-6-nitrospiro[2H-1-benzopyran-2,2' $-(2 \mathrm{H})$-indole] (SP) and its isomers. TD-DFT calculated UV-Vis electronic spectra of the closed and open isomers of this photochromic material are in excellent agreement with the experimental results. Moreover, this paper reports on the results of theoretical investigations of reactivity indices that may govern the conversion between spiropyrans and its isomers. In addition, the solvent and rigidity of the medium significantly control the thermal bleaching of the photogenerated colored isomers and hence the switch ability pattern of the photochromic material. The effect of molecular structure computed by DFT in gas-phase and solvents on $\mathrm{C}_{\text {spiro }}-\mathrm{O}$ bond length has been shown to correlate with photochromic properties. For this compound, DFT optimized geometry could be used to predict photochromism. Furthermore, in an attempt to predict the driving force for $\mathrm{MC} \rightarrow \mathrm{SP}$, this work explores, for the first time, profitable exploitation of the calculated and visualized mapped electrostatic potential energy surfaces (ESP map). Interestingly, it seems that the electrostatic potential forces over the molecular fragments govern spirobond rupture/closure reactions. Thermodynamically, all-trans-colored isomer (CTT) is the most stable merocyaninelike form.
\end{abstract}

\section{Introduction}

Recently, there has been an exponential growth of interest in photochromism for profitable applications in important technologies [1-13]. Spiropyrans are one of the major classes of organic photochromes [2,3]. Its behavior is usually enhanced if a molecule possesses strong electron acceptor substituents such as a nitro group in the 6 and/or 8 positions of its $2 \mathrm{H}$-chromene fragment. Spiropyrans have been extensively studied due to their potential exploitations in several important areas, including optical switching, high-density optical storage, image processing, and display.

The majority of spiropyrans exist in the dark as a neutral closed form that absorbs light only in the ultraviolet region of electromagnetic spectrum. When exposed to UV light, the spiropyran undergoes a molecular rearrangement to produce an open form. The open form of spiropyrans (also called merocyanine (MC)) is colored and shows solvatochromic behavior even in the solid state $[7,12,13]$. The open form of spiropyrans can revert back to the closed form thermally or photochemically by visible light. Thus, the molecules may be switched from the closed to the open form (coloration) with UV light (or thermally in the dark in presence of transition metal ions in polar solvents) and from the open form to the closed form (decoloration) with visible light, or thermally with different time scales' ranges from microseconds to minutes (see Scheme 1). The photochromism of the spiropyran/trans-merocyanine pair has been reviewed previously [3]. The rate and mechanism of the reaction of some spiropyrans and their analogues in 


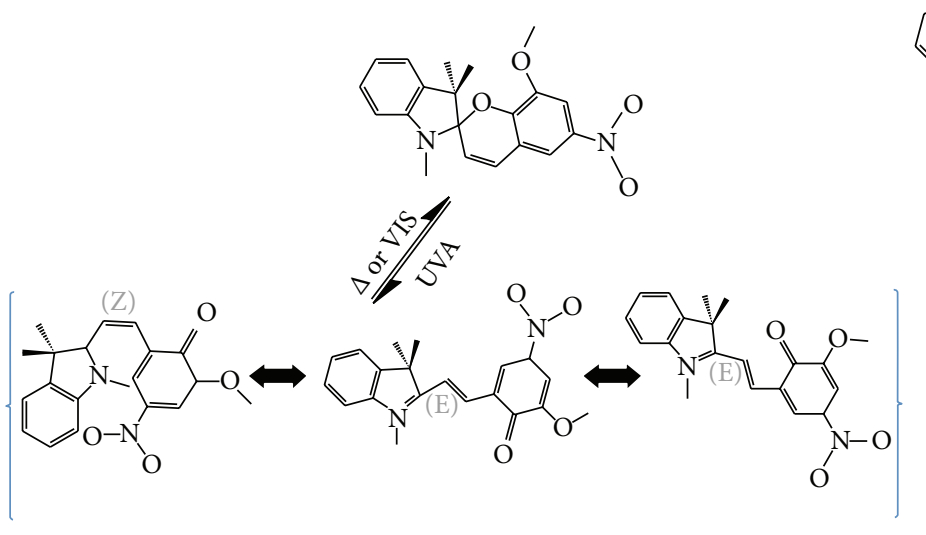

(a)
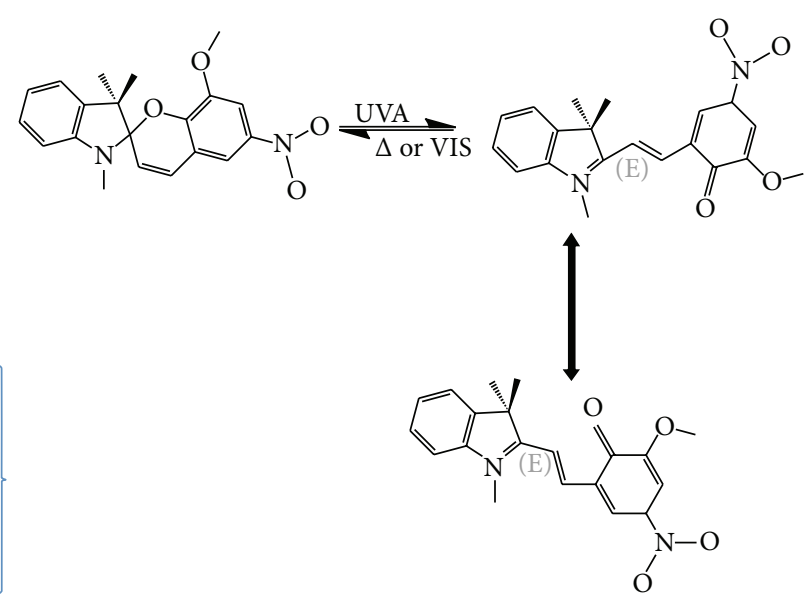

(b)

Scheme 1: Closed and open forms of spiropyran as well as resonance between the open $\mathrm{Z}$ and $\mathrm{E}$ isomers (a). (b) The conversion between the most probable E isomers. Methyl groups are hidden for clarity.

different media have been examined with different spectroscopic techniques [4-14]. Spiropyran photoisomerization was studied using ion mobility-mass spectrometry and three major conformers were identified. Assignment of conformers is based on DFT-B3LYP energy minimized structures and collision cross sections for data collected on light-induced changes in samples. The three conformers were assigned to the spiropyran (SP), a cisoid merocyanine (CCC), and a transoid (TTT) merocyanine. This is the first experimental evidence for a cisoid merocyanine intermediate in spiropyran photoisomerization on a millisecond timescale [15].

Evidence for the TTC form of 6-nitro-BIPS comes from laser desorption/electron diffraction and excited state dynamics [16, 17].

Based on conceptual density functional theory [18], most studies have been conducted on simple model compounds. Fewer theoretical studies of the excited state species have been reported due to computational constrains in the prediction of the excited state properties.

It should be mentioned that the gas-phase dynamics of two classes of photochromic molecules, three spiropyrans and one spirooxazine, have been investigated using both time-resolved mass spectrometry and photoelectron spectroscopy approaches [19]. The comparison of the dynamics of the studied four molecules has been used to propose a sequential photoisomerization mechanism involving four stESP occurring in the first $100 \mathrm{ps}$. The authors have been able to characterize two of these, although questions still remain unanswered for the others.

Previous study [20-22] pointed to the fact that the structure of the photochemically produced colored species, the nature of any reaction intermediates, and the detailed mechanistic processes involved in SP/MC reversible pathways are still unclear (see Scheme 1). Here we try to present the results of a more detailed study about reactivity descriptors and electrostatic potential energy surface maps (ESP maps) that may provide a mechanistic image of the photochemi$\mathrm{cal} /$ thermochemical conversion, which enhances the rational design of new photochromic materials with improved performance. In particular, in this paper the results of TDDFT investigations of the conversion between a spiropyran, namely, $1^{\prime}, 3^{\prime}$-dihydro-8-methoxy-1 $1^{\prime}, 3^{\prime}, 3^{\prime}$-trimethyl-6nitrospiro[2H-1-benzopyran-2, $2^{\prime}-(2 \mathrm{H})$-indole] (SP) and its merocyanines-like isomers (see Scheme 1) on the ground state and the lowest excited state, are reported. Potential energy surfaces and reactivity indices (such as chemical potential, hardness, and softness parameters) developed from HOMO-LUMO frontier orbitals are also presented and discussed. The data computed for electronic singlet states provide a detailed view of the electronic spectroscopy of the isomers of spiropyrans.

\section{Materials and Methods}

2.1. Materials. The spiropyran (SP) $\left(1^{\prime}, 3^{\prime}\right.$-dihydro-8-methoxy-1 $1^{\prime}, 3^{\prime}, 3^{\prime}$-trimethyl-6-nitrospiro[2H-1-benzopyran-2,2' $(2 \mathrm{H})$-indole]) (Sigma-Aldrich, 97\%) was used as received. Pure grade (Aldrich) solvents were used.

2.2. Instruments. UV-Vis absorption spectra were measured in the range of $250-800 \mathrm{~nm}$ using diode array ocean optics spectrometer with spectra suite operating software. The source of UV-Vis irradiation is a homemade photoirradiation apparatus with an 8 Watt UVA lamp.

2.3. General Procedure for the SP Photochemical Reactions. The color developing reaction in a quartz cell is carried out using 8 Watt UVA lamp and monitored spectrophotometrically. The color fading up reaction monitored in the dark using the repeat scan mode of Spectra Suite Ocean Optics software. All the measurements were carried out at room temperature $\approx 22^{\circ} \mathrm{C}$. 


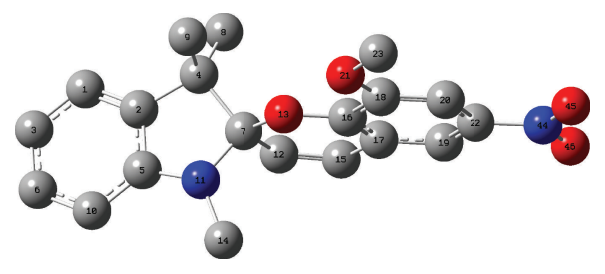

(a)

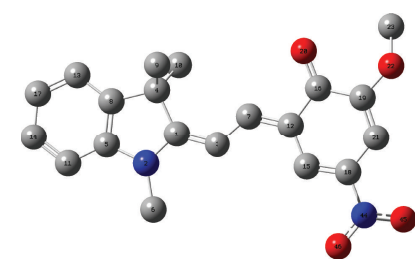

(b)

FIGURE 1: (a) The optimized geometry of the colorless SP structure ( $\mathrm{H}$ atoms are hidden for clarity) and (b) the optimized geometry of the TTT isomer (see Supplementary Materials (Tables 1 and 2), available online at http://dx.doi.org/10.1155/2016/6765805, for detailed optimized geometry parameters of the SP/MC isomers in gas-phase and in water, ethanol, and toluene).

2.4. Theoretical Computations. The theoretical calculations are carried out using Gaussian 09, version D (64-Bit, Linda), Gaussian Inc. (USA), and Spartan'14 parallel 64-bit version (Wavefunction Inc., USA) quantum chemical packages within the framework of DFT and TD-DFT (with $6 \mathrm{MOs}$ taken into account in the $\mathrm{CI}$ ) and visualized by Gaussview 05 program (in case of Gaussian 09 computations). 6and 12 -core pro-MAC computer were used to perform the computations. The geometry optimization of closed and open forms of the photochromic dye was carried out in vacuum as implemented in the Gaussian 09 package [17]. The geometry of the dye was optimized using B3LYP [23] functional with the 6-21G-basis set, which is a good compromise between accuracy and efficiency. The further expansion of the basis set such as using 6-31+G(d) has less impact on the accuracy of the molecular parameters. Attempts with other basis sets result in no noticeable changes. However, using 6-311G* basis set improves the UV-Vis absorption spectral parameters $(\lambda$ and oscillator strengths). Potential energy surfaces are calculated using B3LYP with $6-311 \mathrm{G}^{*}$ basis set. First excited state $\left(\mathrm{S}_{1}\right)$ calculations are obtained at Configuration Interaction Singles (CIS) level with similar basis sets $\left(6-311 G^{*}\right)$.

DataGraph 3.2 (Visual Data Tools, Inc., USA) software was used for the graphical representation of the TD-DFT UVVis electronic spectra.

The DFT-B3LYP method has been demonstrated to predict excellent geometries and energies. In addition, this level of computation was found to take into account different geometrical orientations of merocyanine-like isomers. Vibration frequency calculations were performed to ensure geometry optimization with minimum energy structure. The UV-Vis spectrum was also studied in gas-phase as well as in two representative solvents (polar protic ethanol and nonpolar toluene). The useful reaction field model used for solvation is the conductor polarized continuum model (CPCM) [24].

\section{Results and Discussion}

3.1. The SP/MC Conversion. Irradiation in the UV region leads to the cleavage of the $\mathrm{C}_{\text {spiro }}-\mathrm{O}$ bond resulting in different isomers or conformers. Figure 1 shows the optimized geometry of the SP closed form and one of its colored MC isomer.

It is suggested that irradiation results in the cis-MC (Zusammen, $\mathrm{Z}$ isomer), which dominantly exist in the more stable TTT form (see Scheme 1(a)). The rotation about the central C-C bonds in cis-MC yields trans-MC (Scheme 1(a)). The MC product is a hybrid of different CCC, CCT, TTC, and TTT forms. However, it seems plausible to consider the direct ring opening through $\mathrm{C}_{\text {spiro }}-\mathrm{O}$ bond rupture to yield the energetically apparent stable $\mathrm{Z}$ isomer as represented in Scheme 1(b).

The $\mathrm{MC} \rightarrow$ SP reverse isomerization usually occurs spontaneously and can be accelerated thermally or by visible light $[2,3,19,20]$. Spiropyrans are also capable of isomerizing in the presence of metal ions even under dark conditions, since the merocyanine form is stabilized by coordination to the metal ions $[3,11,22]$. The main problem of spiropyrans is their low stability and for this reason several attempts have been performed in order to incorporate or disperse them into a polymer matrix [25]. This results in delayed color fading of the MC-like isomers, which accompanies the dark conversion to the closed isomer (see Scheme 1). Specific interactions between the polymer functional groups and the MC-like forms as well as polymer free volume available for the isomerization are the obvious reasons for the noticed delay of MC-like isomers to revert back to SP form. The dark fading is also retarded in presence of transition metal ions such as samarium ions due to complexation with the chelating groups of the MC-like isomers. Consequently, the number of switching cycles should be decreased significantly. Subsequently, inclusion of closed SP in PMMA thin film or the presence of a lanthanide ion significantly stabilizes the photogenerated TTT isomer [26, 27].

To explore the relationship between photochromism and structural parameters such as particular bond lengths [20,28] for this material, within the framework of the DFT, we studied the molecular geometry of the closed and open forms in the gas-phase. Upon the spiropyran-merocyanine transformation the $\mathrm{C}_{7}$ atom $\left(\mathrm{C}_{\text {spiro }}\right.$ see Figure $\left.1(\mathrm{a})\right)$ changed from $\mathrm{sp}^{3}$ hybridized to $\mathrm{sp}^{2}$ hybridized and the two aromatic moieties of the molecule changed from being perpendicular and became coplanar substantially. Generally, when compared to gas-phase calculations, the solvent environment alters the charge distribution of molecules. This is reflected in bond length change. Bond length $\mathrm{C}_{\text {spiro }}-\mathrm{O}$ increases noticeably in the order: gas-phase $<$ toluene $<$ ethanol $<$ water reflecting easier bond breaking in polar solvent. Moreover, the localized positive/negative Mulliken [29] charge on the $\mathrm{C}_{\text {spiro }}^{\delta+}-\mathrm{O}^{\delta-}$ atoms decreases slightly in the reverse order leading to a relatively less electrostatic attraction between $\mathrm{C}_{\text {spiro }}-\mathrm{O}$ atoms. 
TABLE 1: HOMO/LUMO energies, chemical potential, hardness, and softness of SP/MC isomers.

\begin{tabular}{|c|c|c|c|c|c|c|}
\hline \multirow{2}{*}{$\begin{array}{l}\text { Reactivity indices calculated from HOMO/LUMO } \\
\text { energies (a.u.) }\end{array}$} & \multicolumn{3}{|c|}{ SP closed form } & \multicolumn{3}{|c|}{ Merocyanine-like isomers } \\
\hline & Gas-phase & Toluene & $\mathrm{EtOH}$ & $\mathrm{CCC}$ & TTC & TTT \\
\hline$\epsilon_{\text {LUMO }}$ & -0.07782 & -0.08822 & -0.08812 & -0.10667 & -0.10023 & -0.10994 \\
\hline$\epsilon_{\mathrm{HOMO}}$ & -0.19082 & -0.1925 & -0.19290 & -0.19487 & -0.19139 & -0.19217 \\
\hline$\mu$ & -0.1343 & -0.1404 & -0.1405 & -0.15077 & -0.14581 & -0.15101 \\
\hline$\eta$ & +0.1130 & +0.1044 & +0.1047 & +0.0882 & +0.09116 & +0.08223 \\
\hline$S,(\text { a.u. })^{-1}$ & 8.8496 & 9.5785 & 9.5511 & 11.3379 & 10.9697 & 12.1610 \\
\hline
\end{tabular}

This synergistically with the bond length favors easier bond breaking in more polar medium (Online Resources: see Table 1; $\mathrm{C}_{7}$ and $\mathrm{O}_{13}$ ). Moreover, based on the larger Mulliken negative charge values of the substituted benzo ketone moiety, it could be easily predicted that the ketonic $\mathrm{O}$ as well as the methoxy $\mathrm{O}$ atoms has larger abilities to coordinate with a transition or a lanthanide metal positive ion in both SP/MC forms.

See Supplementary Tables 1 and 2 (Online Resources): Supplementary Table 1 shows parameters of the optimized geometries and Mulliken charges of SP in different media (refer to Figure 1(a) for atom tags, bond connectivity, and angles); Supplementary Table 2 shows parameters of the optimized geometries and Mulliken charges of the different MC isomers (refer to Scheme 1(a) and Figure 1(b)).

It is noteworthy to mention that in the application of quantum mechanical calculations to molecular systems, the calculation of effective atomic charge plays an important role in such cases. Mulliken atomic charges are calculated by determining the electron population of each atom as defined by the basis function.

\subsection{DFT Local Indices for Reactivity and Liability of Electron} Density. Local properties are highly desirable in establishing a reactivity-oriented description of molecular systems [3034].

Global reactivity indices were estimated according to the equations recommended by Parr and Yang [30, 31]. In particular, the electronic chemical potentials $(\mu)$, chemical hardness $(\eta)$, and softness $(S=1 / \eta)$ of the SP/MC isomers studied were evaluated in terms of the one-electron energies of the frontier molecular orbital HOMO and LUMO, using the following equations:

$$
\begin{aligned}
& \mu=-\frac{1}{2}(I+A) \approx \frac{1}{2}\left(\epsilon_{\mathrm{LUMO}}+\epsilon_{\mathrm{HOMO}}\right), \\
& \eta=(I-A) \approx\left(\epsilon_{\mathrm{LUMO}}-\epsilon_{\mathrm{HOMO}}\right)
\end{aligned}
$$

where $\epsilon_{\mathrm{HOMO}}$ and $\epsilon_{\mathrm{LUMO}}$ are the energies of the highest occupied and the lowest unoccupied molecular orbitals, HOMO and LUMO, respectively. The energies of KohnSham frontier molecular orbital have been used to calculate reactivity descriptors coming from conceptual DFT [30-33].

The chemical potential $\mu$ measures the escaping tendency of electron from equilibrium and the global hardness $\eta$ can be seen as the resistance to charge transfer (or the band gap), while softness $S$ gives indication of how large is electron transfer to/from the molecule when the chemical potential changes.

The knowledge of reactivity on a molecule is of a crucial interest because it allows understanding interactions that are operating during a reaction mechanism. In particular electrostatic interactions have been successfully explained by the use of the molecular electrostatic potential [18, 3436]. The calculated values are tabulated in Table 1. Escaping tendency of electronic charge $\mu$-value increases by increasing the polarity of the solvent and/or by $\mathrm{C}_{\text {spiro }}-\mathrm{O}$ bond cleavage to form MC isomers. Also, resistance to charge transfer measured by $\eta$ decreases and, thus, softness of molecules increases. It indicates larger charge transfer from/to the MC relative to SP, following the order

$$
\begin{aligned}
\mathrm{SP}(\text { gas-phase }) & <\mathrm{SP}(\mathrm{EtOH}) \leq \mathrm{SP}(\text { Toluene })<\mathrm{TTC} \\
& <\mathrm{MCCT}<\mathrm{TTT}
\end{aligned}
$$

(see Table 1). The calculated reactivity descriptors listed in Table 1 favor largest charge transfer liability in case of TTT isomer. This is in agreement with findings of a recent study of photoisomerization among ring-open merocyanines by reaction dynamics and wave packet oscillations induced by tunable femtosecond pulses [35].

3.3. UV Irradiation and Thermal Relaxation. Scheme 1(a) describes collectively the spectral observations of liquidphase experiments depicted in Figure 2 (bathochromic shift of SP peak forming isosbestic point as the time of irradiation increases leading to large enhancement of the color of the MC-like isomers generated).

Our spectra calculations (Table 2) show a reaction pathway represented by the following scheme: SP $\rightarrow$ CCC $\rightarrow$ TTC $\rightarrow$ TTT (see Figure 2(a)). This is in agreement with the results obtained above, which shows that the calculated reactivity descriptors listed in Table 1 favor largest charge transfer liability in case of TTT isomer. This is reflected in its red shifted longest wavelength absorption peak relative to other MC isomers.

Some representative molecular orbitals involved in the electronic transitions are shown in Figure 3. In case of SP closed form, it is obvious that electron transfer transition between HOMO and LUMO reflects the localized nature of the donor (indole) and acceptor (nitro benzene derivative) parts of the two perpendicular moieties of the molecules; see Figure 3. Some representative molecular orbitals (MOs) involved in the electronic transitions listed in Table 2 for 
TABLE 2: Computed spectroscopic characteristics of the SP/MC isomers and MOs involved in the CI transitions.

\begin{tabular}{|c|c|c|c|c|}
\hline \multirow{3}{*}{ Excited state } & Closed form (SP) & Open form (CCC) & Open form (TTC) & Open form (TTT) \\
\hline & $\lambda, \mathrm{eV}, \mathrm{nm}(f)$ & $\lambda, \mathrm{eV}, \mathrm{nm}(f)$ & $\lambda, \mathrm{eV}, \mathrm{nm}(f)$ & $\lambda, \mathrm{eV}, \mathrm{nm}(f)$ \\
\hline & MOs involved & MOs involved & MOs involved & MOs involved \\
\hline \multirow{3}{*}{1} & $2.672,464.0(0.011)$ & $1.737,713.9(0.075)$ & $1.716,722.4(0.029)$ & $1.805,686.9(0.039)$ \\
\hline & $93 \rightarrow 94$ & $93 \rightarrow 94$ & $92 \rightarrow 94$ & $92 \rightarrow 94$ \\
\hline & & $93 \rightarrow 95$ & $93 \rightarrow 94$ & $93 \rightarrow 94$ \\
\hline \multirow{5}{*}{2} & $3.483,356.0(0.025)$ & $2.252,550.5(0.031)$ & $2.096,591.5(0.234)$ & $2.015,615.2(0.245)$ \\
\hline & $93 \rightarrow 95$ & $91 \rightarrow 94$ & $92 \rightarrow 94$ & $92 \rightarrow 94$ \\
\hline & & $92 \rightarrow 94$ & $93 \rightarrow 94$ & $93 \rightarrow 94$ \\
\hline & & $93 \rightarrow 94$ & $93 \rightarrow 95$ & $93 \rightarrow 96$ \\
\hline & & $93 \rightarrow 95$ & & \\
\hline \multirow{4}{*}{3} & $3.594,345.01(0.0001)$ & $2.474,501.2(0.364)$ & $2.378,521.2(0.004)$ & $2.267,546.8(0.008)$ \\
\hline & $89 \rightarrow 94$ & $92 \rightarrow 94$ & $90 \rightarrow 94$ & $90 \rightarrow 94$ \\
\hline & & $93 \rightarrow 94$ & $91 \rightarrow 94$ & $91 \rightarrow 94$ \\
\hline & & $93 \rightarrow 95$ & & \\
\hline \multirow{6}{*}{4} & $3.691,335.9(0.194)$ & $2.778,446.3(0.017)$ & $2.806,441.7(0.152)$ & $2.716,456.4(0.117)$ \\
\hline & $91 \rightarrow 94$ & $91 \rightarrow 94$ & $90 \rightarrow 94$ & $90 \rightarrow 94$ \\
\hline & $92 \rightarrow 94$ & $92 \rightarrow 94$ & $92 \rightarrow 95$ & $93 \rightarrow 94$ \\
\hline & & $92 \rightarrow 95$ & $92 \rightarrow 96$ & $93 \rightarrow 95$ \\
\hline & & & $93 \rightarrow 94$ & $93 \rightarrow 96$ \\
\hline & & & $93 \rightarrow 95$ & \\
\hline \multirow{5}{*}{5} & $3.850,321.9(0.036)$ & $3.056,405.7(0.011)$ & $2.810,441.2(0.004)$ & $2.782,445.6(0.034)$ \\
\hline & $90 \rightarrow 94$ & $90 \rightarrow 94$ & $90 \rightarrow 94$ & $90 \rightarrow 94$ \\
\hline & $91 \rightarrow 94$ & $90 \rightarrow 95$ & $91 \rightarrow 94$ & $91 \rightarrow 94$ \\
\hline & $92 \rightarrow 94$ & $91 \rightarrow 94$ & $92 \rightarrow 94$ & $92 \rightarrow 96$ \\
\hline & $92 \rightarrow 95$ & $92 \rightarrow 95$ & $92 \rightarrow 95$ & $93 \rightarrow 96$ \\
\hline
\end{tabular}

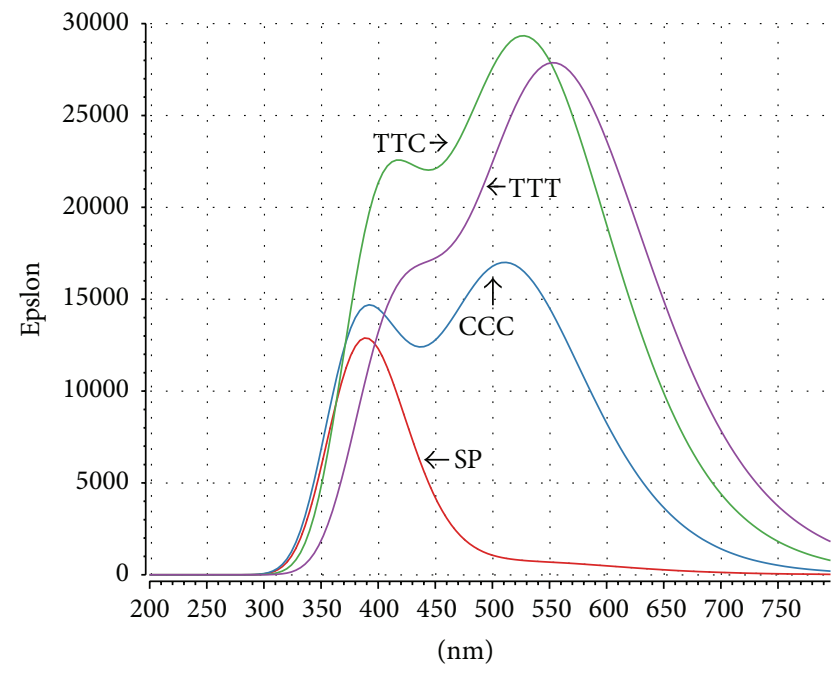

(a)

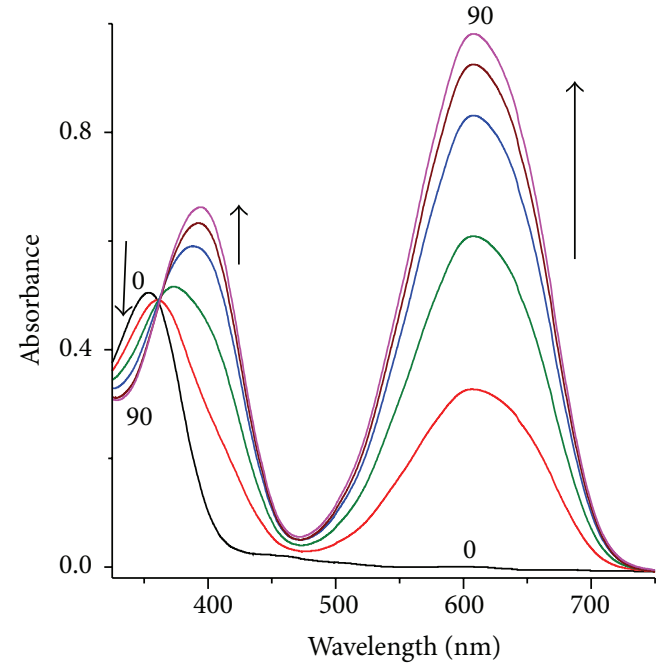

(b)

FIGURE 2: (a) Theoretically computed electronic spectra of the SP (red)/MC [CCC (cyan), TTC (green), and TTT(purple)] isomers. (b) Experimentally determined photo-induced transformation $\left(\lambda_{\text {irradiation }}=365 \mathrm{~nm}\right.$ on the absorption spectrum of $5 \times 10^{-5} \mathrm{M}$ of SP in toluene). Time of irradiation from the bottom $=0,10,20,30,40,60,90 \mathrm{sec}$. The sharp isosbestic point in (b) reveals the isomerization reaction between a closed SP and an open merocyanine form. 


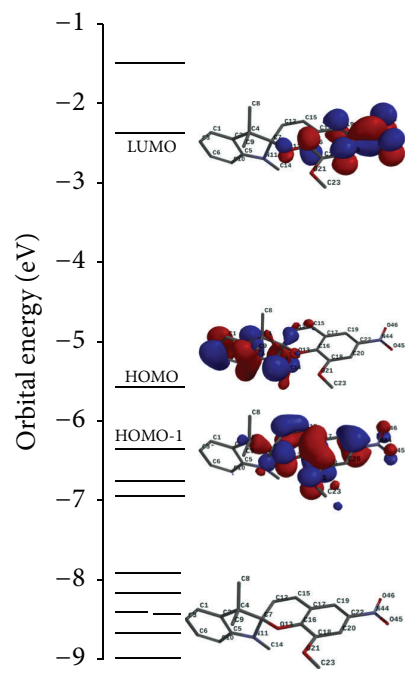

SP closed form

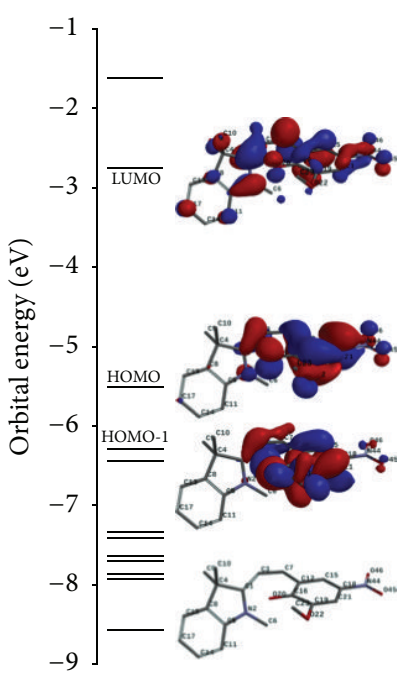

CCC opened all cisoid form

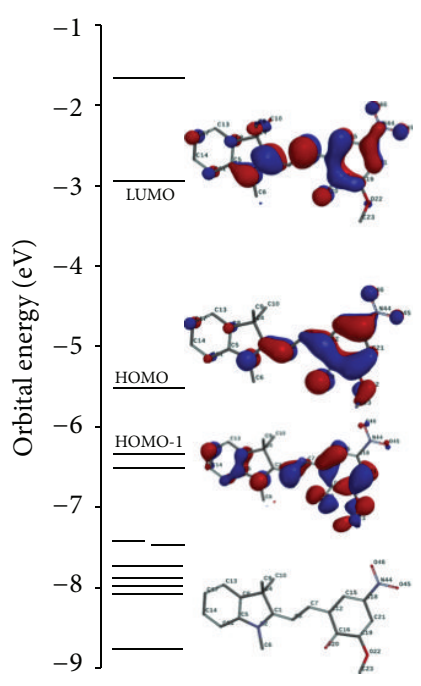

TTC opened form

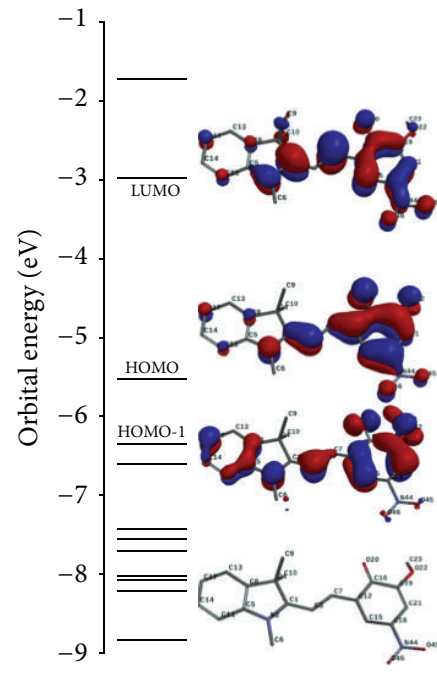

TTT opened form

FIGURE 3: Orbital energies and some MOs involved in the electronic transitions of different isomers.

\begin{tabular}{|c|c|c|}
\hline SP form & $\stackrel{h v}{\longrightarrow}$ & $\mathrm{S}_{1}$ excited state $\mathrm{SP}^{*}$ \\
\hline ESP map is of PE ranges from -169 to & & ESP map of PE ranges from -193 \\
\hline $\begin{array}{c}+116 \mathrm{kJmol}^{-1} ; \text { difference }=43 \mathrm{kJmol}^{-1} \\
\quad \text { Mulliken B.O. of } \mathrm{C}_{\text {spiro }}-\mathrm{O}=0.90\end{array}$ & & $\begin{array}{c}\text { to }+126 \text {; the difference }=67 \mathrm{kJmol}^{-1} \\
\mathrm{C}_{\text {spiro }}-\mathrm{O} \mathrm{BO}{ }^{*}=0.83\end{array}$ \\
\hline
\end{tabular}

SCHEME 2

SP/TTT isomer and some are depicted graphically in Figure 3.

It should be pointed out that previous quantum chemical calculations have shown that TTC is the most stable and TTT is the second most stable [20,37-41]. However, several computational studies used a reduced atom set to facilitate computations making comparison to the parent system more difficult.

3.4. Analysis of Molecular Electrostatic Surface Potential (ESP). The electrostatic surface potential (ESP) provides a visual method to understand the relative polarity of the compounds [34,36]. Mapped electrostatic surfaces potential (ESP map) are shown in Figure 4 which illustrates the 3D charge distributions of the molecule. The ESP map is a plot of electrostatic potential, mapped onto the constant (HOMO) electron density or total electron density surface. One of the main purposes of finding the electrostatic potential is to find the reactive site of a molecule. The importance of which lies in the fact that it simultaneously displays molecular size, shape, and positive, negative, and neutral electrostatic potential energy regions in terms of color grading (Figure 4) and is very useful in molecular structure-physiochemical property relationship [22, 42, 43]. Knowledge of the charge distributions can be used to determine how molecules interact with one another. In molecular ESP maps, the negative electrostatic potentials are shown in red, the intensity of which is proportional to the absolute value of the potential energy, and positive electrostatic potentials are shown in blue while green indicates surface areas where the potentials are close to zero. These surfaces are computed at the 0.0004 a.u. isodensity surface. Potential increases are in the following order: red $<$ orange $<$ yellow $<$ green $<$ blue (Figure 4 ). The figure provides a visual representation of the chemically active sites and comparative reactivity of atoms. In SP/MC, the blue indicates the strongest attraction and red indicates the strongest repulsion. Regions of negative value are usually associated with the lone pair of electronegative atoms. As can be seen from the ESP map of the studied molecules (Figure 4), while regions having the negative potential are over the indole part of the SP the regions having the zero or small positive potential are over the aromatic moiety that has methoxy and nitro group, especially on the $\mathrm{C}_{\text {spiro }}-\mathrm{O}$. It is interesting to note that negative and positive potentials according to the ESP map are uniformly distributed over the whole TTT molecule. Referring to Figure 4, it could be seen that upon light excitation the potential energy rises as Scheme 2.

Consequently, $\mathrm{C}_{\text {spiro }}-\mathrm{O}$ bond rupture occurs easily due to the increase in $\mathrm{PE}$ and the decrease in $\mathrm{C}_{\text {spiro }}-\mathrm{O}$ bond order within femtosecond time domain [19] generating the CCC form, which is characterized by ESP map with potential energy values range between -207 and $+141 \mathrm{kJmol}^{-1}$ of $66 \mathrm{kJmol}^{-1}$ difference. Isomerization to TTC (of ESP map; -200 to $+176 \mathrm{kJmol}^{-1}$; difference $=34 \mathrm{kJmol}^{-1}$ ) occurs with subsequent fast conversion around the bonds of the bridge 


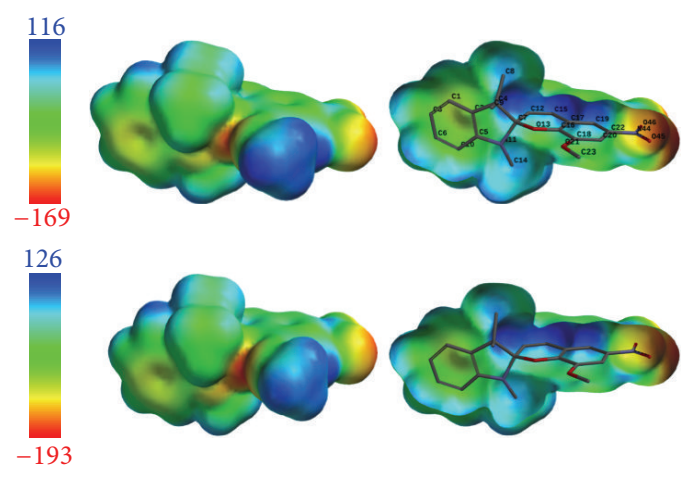

SP ESP map (potential ranges from -169 to $+116 \mathrm{kJmol}^{-1}$ ) Difference $=43 \mathrm{kJmol}^{-1}$ and below is $\mathrm{SP}$ in $\mathrm{S}_{1}$ state $\mathrm{PE}$ ranges from -193 to +126 and the difference $=67 \mathrm{kJmol}^{-1}$

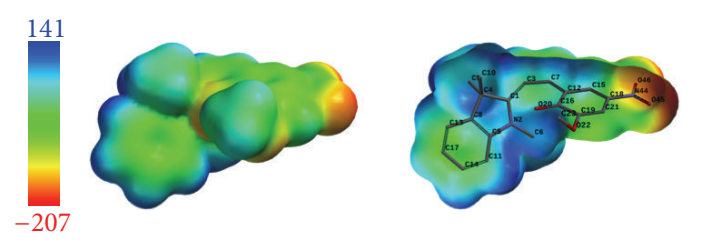

CCC ESP map (potential ranges from -207 to $+141 \mathrm{kJmol}^{-1}$ ) Difference $=66 \mathrm{kJmol}^{-1}$
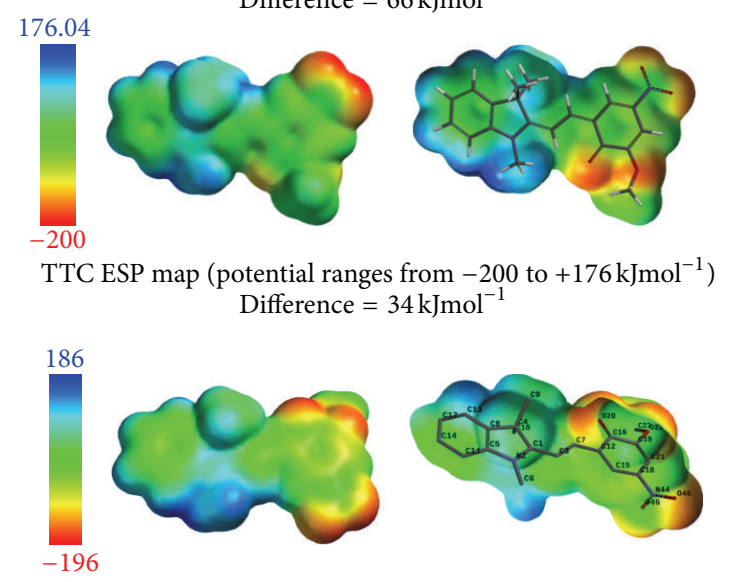

TTT ESP map (potential ranges from -196 to $+186 \mathrm{kJmol}^{-1}$ ) Difference $=10 \mathrm{kJmol}^{-1}$

FIGURE 4: The molecular electrostatic potential mapped onto total SCF electron density for the different forms of closed SP and merocyanine-like isomer (isovalue for the isosurfaces) with different color codes from -ve value (red) to +ve value (blue) expressed in $\mathrm{kJmol}^{-1}$.

to the most stable TTT $\left(-196\right.$ to $+186 \mathrm{kJmol}^{-1}$; difference $=$ $10 \mathrm{kJmol}^{-1}$ ), which has, among other forms, the smallest difference between the maximum and minimum potential energy value of $10 \mathrm{kJmol}^{-1}$.

ESP maps clearly distinguish the electrostatic potentials over the different molecular isomers and could be used to explain the driving force for ring closer of TTT and other open form isomers. ESP maps point to the spreading of lowest attractive/repulsive energy over the SP form suggesting a visualizing way to explain driving force for the reverse dark reaction $\mathrm{CCC} \leftrightarrow \mathrm{TTC} \leftrightarrow \mathrm{TTT} \rightarrow \mathrm{SP}$.

\section{Conclusions}

The results reveal that the photo-induced ring opening process of the SP closed form generates three possible planar merocyanine-like (MC) colored isomers (CCC, TTC, and TTT). This paper tackled the issue of the photo-induced isomers of SP in a new way using new theoretical approach. This is done via considering results of reactivity descriptors and ESP map.

The calculated reactivity descriptors such as chemical potential, hardness, and softness of the photochromic material and its open forms isomers favor the largest charge transfer liability in case of TTT isomer. This finding also correlates with Mulliken charges and TD-DFT calculated and experimentally measured electronic spectra.

The effect of molecular structure generated by DFT in gas-phase and solvents on calculated $\mathrm{C}_{\text {spiro }}-\mathrm{O}$ bond length has been shown to correlate with photochromic properties. For this compound, DFT optimized geometry could be used to predict the absorption wavelength of the colored photochromic material. The larger the $\mathrm{C}_{\text {spiro }}-\mathrm{O}$ bond distance is, the longer the absorption wavelength is.

Favorable SP ESP map of lowest energy limits relative to other open forms encourages bleaching of the photogenerated open colored MC forms, suggesting a visualizing way to explain driving force for the reverse pathway CCC $\leftrightarrow$ TTC $\leftrightarrow$ $\mathrm{TTT} \rightarrow$ SP.

The detailed theoretically obtained knowledge (such as reactivity descriptors derived from HOMO-LUMO energies and ESP maps) should result in considering a set of new parameters that are important guidance for predicting new photochromic materials with improved performance.

\section{Competing Interests}

The authors declare that there is no conflict of interests regarding the publication of this paper.

\section{References}

[1] R. C. Bertelson, "Photochromic processes involving heterolytic cleavage," in Techniques of Chemistry, G. H. Brown, Ed., vol. 3, pp. 45-433, Wiley-Interscience, New York, NY, USA, 1971.

[2] H. Duerr and H. Bouas-Laurent, Photochromism: Molecules and Systems, Elsevier, New York, NY, USA, 1990.

[3] G. Berkovic, V. Krongauz, and V. Weiss, "Spiropyrans and spirooxazines for memories and Switches," Chemical Reviews, vol. 100, no. 5, pp. 1741-1753, 2000.

[4] R. Guglielmetti, "Chapter 8-4n+2 systems: spiropyrans," in Photochromism: Molecules and Systems, H. Durr and H. BouasLaurent, Eds., pp. 314-466, Elsevier, Amsterdam, Netherlands, 1990.

[5] R. Guglielmetti, "Chapter 23-spiropyrans and related compounds," in Photochromism: Molecules and Systems, H. Durr and H. Bouas-Laurent, Eds., pp. 855-878, Elsevier, Amsterdam, Netherlands, 1990.

[6] M. S. Attia, M. H. Khalil, M. S. A. Abdel-Mottaleb, M. B. Lukyanova, Yu. A. Alekseenko, and B. Lukyanov, "Effect of complexation with lanthanide metal ions on the photochromism 
of (1,3,3-trimethyl-5' -hydroxy-6' -formyl- indoline-spiro2,2' [2h]chromene) in different media," International Journal of Photoenergy, vol. 2006, Article ID 42846, 9 pages, 2006.

[7] B. S. Lukyanov, A. V. Metelitsa, N. A. Voloshin et al., "Solid state photochromism of spiropyrans," International Journal of Photoenergy, vol. 7, no. 1, pp. 17-22, 2005.

[8] J. Berthet, S. Delbaere, V. Lokshin, A. Samat, J. C. Micheau, and G. Vermeersch, "NMR studies of the polyphotochromic behaviour of biphotochromic compounds," International Journal of Photoenergy, vol. 6, no. 4, pp. 215-220, 2004.

[9] S. Delbaere, J. C. Micheau, J. Berthet, and G. Vermeersch, "Contribution of NMR spectroscopy to the mechanistic understanding of photochromism," International Journal of Photoenergy, vol. 6, no. 4, pp. 151-158, 2004.

[10] A. O. Bulanov, L. D. Popov, I. N. Shcherbakov et al., "Synthesis, IR, UV/vis-, ${ }^{1} \mathrm{H}$ NMR and DFT study of chelatophore functionalized 1,3-benzoxazinone spiropyrans," Spectrochimica ActaPart A: Molecular and Biomolecular Spectroscopy, vol. 71, no. 3, pp. 1146-1152, 2008.

[11] E. Bakeir, G. M. Attia, M. Lukyanova, B. Lukyanov, and M. S. A. Abdel-Mottaleb, "The effect of $\mathrm{Tb}$ and $\mathrm{Sm}$ ions on the photochromic behavior of two spiropyrans of benzoxazine series in solution," Research Letters in Physical Chemistry, vol. 2008, Article ID 314898, 4 pages, 2008.

[12] K. Kinashi, S. Nakamura, Y. Ono, K. Ishida, and Y. Ueda, "Reverse photochromism of spiropyran in silica," Journal of Photochemistry and Photobiology A: Chemistry, vol. 213, no. 2-3, pp. 136-140, 2010.

[13] F. Zhang, X. Zou, W. Feng et al., "Microwave-assisted crystallization inclusion of spiropyran molecules in indium trimesate films with antidromic reversible photochromism," Journal of Materials Chemistry, vol. 22, no. 48, pp. 25019-25026, 2012.

[14] M. Campredon, R. Guglielmetti, A. Samat, and A. Alberti, "ESR studies on some spiropyrans, spironaphthropyrans, and spirooxazines," The Journal of Chemical Physics, vol. 91, no. 1112, pp. 1830-1836, 1830.

[15] R. A. Rogers, A. R. Rodier, J. A. Stanley, N. A. Douglas, X. Li, and W. J. Brittain, "A study of the spiropyran-merocyanine system using ion mobility-mass spectrometry: experimental support for the cisoid conformation," Chemical Communications, vol. 50, no. 26, pp. 3424-3426, 2014.

[16] A. Gahlmann, I.-R. Lee, and A. H. Zewail, "Direct structural determination of conformations of photoswitchable molecules by laser desorption-electron diffraction," Angewandte ChemieInternational Edition, vol. 49, no. 37, pp. 6524-6527, 2010.

[17] C. J. Wohl and D. Kuciauskas, "Excited-state dynamics of spiropyran-derived merocyanine isomers," The Journal of Physical Chemistry B, vol. 109, no. 47, pp. 22186-22191, 2005.

[18] P. Geerlings, F. de Proft, and W. Langenaeker, "Conceptual density functional theory," Chemical Reviews, vol.103, no. 5, pp. 1793-1873, 2003.

[19] L. Poisson, K. D. Raffael, B. Soep, J.-M. Mestdagh, and G. Buntinx, "Gas-phase dynamics of spiropyran and spirooxazine molecules," Journal of the American Chemical Society, vol. 128, no. 10, pp. 3169-3178, 2006.

[20] Y. Sheng, J. Leszczynski, A. A. Garcia, R. Rosario, D. Gust, and J. Springer, "Comprehensive theoretical study of the conversion reactions of spiropyrans: substituent and solvent effects," The Journal of Physical Chemistry B, vol.108, no. 41, pp. 16233-16243, 2004.
[21] A.-K. Holm, O. F. Mohammed, M. Rini, E. Mukhtar, E. T. J. Nibbering, and H. Fidder, "Sequential merocyanine product isomerization following femtosecond UV excitation of a spiropyran," Journal of Physical Chemistry A, vol. 109, no. 40, pp. 8962-8968, 2005.

[22] V. I. Minkin, A. V. Metelitsa, I. V. Dorogan, B. S. Lukyanov, S. O. Besugliy, and J.-C. Micheau, "Spectroscopic and theoretical evidence for the elusive intermediate of the photoinitiated and thermal rearrangements of photochromic spiropyrans," Journal of Physical Chemistry A, vol. 109, no. 42, pp. 9605-9616, 2005.

[23] M. J. Frisch, G. W. Trucks, H. B. Schlegel et al., GAUSSIAN 09, Revision D.01, Gaussian Inc, Wallingford, Conn, USA, 2013.

[24] J. Tomasi, B. Mennucci, and R. Cammi, "Quantum mechanical continuum solvation models," Chemical Reviews, vol. 105, no. 8, pp. 2999-3093, 2005.

[25] R. Klajn, "Spiropyran-based dynamic materials," Royal Society of Chemistry, vol. 43, no. 17, pp. 1-488, 2014.

[26] A. Samoladas, D. Bikiaris, T. Zorba, K. M. Paraskevopoulos, and A. Jannakoudakis, "Photochromic behavior of spiropyran in polystyrene and polycaprolactone thin films-effect of UV absorber and antioxidant compound," Dyes and Pigments, vol. 76, no. 2, pp. 386-393, 2008.

[27] S. N. Ali, Photochromism and spectroscopic studies of some spiropyran complexes of some transition metal ions [M.S. thesis], Faculty of Science, Ain Shams University, Cairo, Egypt, 2015.

[28] S. Kumar, K. Velasco, and A. McCurdy, "X-ray, kinetics and DFT studies of photochromic substituted benzothiazolinic spiropyrans," Journal of Molecular Structure, vol. 968, no. 1-3, pp. 13-18, 2010.

[29] R. S. Mulliken, "A new electroaffinity scale; Together with data on valence states and on valence ionization potentials and electron affinities," The Journal of Chemical Physics, vol. 2, no. 11, pp. 782-793, 1934.

[30] J. Hobley and V. Malatesta, "Energy barrier to TTC-TTT isomerisation for the merocyanine of a photochromic spiropyran," Physical Chemistry Chemical Physics, vol. 2, no. 1, pp. 57-59, 1989.

[31] R. G. Parr and W. Yang, "Density functional approach to the frontier-electron theory of chemical reactivity," Journal of the American Chemical Society, vol. 106, no. 14, pp. 4049-4050, 1984.

[32] P. W. Ayers, J. S. M. Anderson, and L. J. Bartolotti, "Perturbative perspectives on the chemical reaction prediction problem," International Journal of Quantum Chemistry, vol. 101, no. 5, pp. 520-534, 2005.

[33] F. Zielinski, V. Tognetti, and L. Joubert, "Condensed descriptors for reactivity: a methodological study," Chemical Physics Letters, vol. 527, pp. 67-72, 2012.

[34] A. Savin, C. J. Umrigar, and X. Gonze, "Relationship of KohnSham eigenvalues to excitation energies," Chemical Physics Letters, vol. 288, no. 2-4, pp. 391-395, 1998.

[35] P. Politzer and J. S. Murray, "The fundamental nature and role of the electrostatic potential in atoms and molecules," Theoretical Chemistry Accounts, vol. 108, no. 3, pp. 134-142, 2002.

[36] J. S. Murray and P. Politzer, "The electrostatic potential: an overview," Wiley Interdisciplinary Reviews: Computational Molecular Science, vol. 1, no. 2, pp. 153-163, 2011.

[37] Y. Futami, M. Lim, L. S. Chin, S. Kudoh, M. Takayanagi, and M. Nakata, "Conformations of nitro-substituted spiropyran and merocyanine studied by low-temperature matrix-isolation 
infrared spectroscopy and density-functional-theory calculation," Chemical Physics Letters, vol. 370, no. 3-4, pp. 460-468, 2003.

[38] J. Hobley, U. Pfeifer-Fukumura, M. Bletz, T. Asahi, H. Masuhara, and H. Fukumura, "Ultrafast photo-dynamics of a reversible photochromic spiropyran," The Journal of Physical Chemistry A, vol. 106, pp. 2265-2270, 2002.

[39] J. Hobley and V. Malatesta, Physical Chemistry Chemical Physics, 2000.

[40] J. Hobley, V. Malatesta, R. Millini, L. Montanari, and W. O Neil Parker Jr., "Proton exchange and isomerisation reactions of photochromic and reverse photochromic spiro-pyrans and their merocyanine forms," Physical Chemistry Chemical Physics, vol. 1, no. 14, pp. 3259-3267, 1999.

[41] J. Hobley, V. Malatesta, W. Giroldini, and W. Stringo, " $\pi$-Cloud and non-bonding or $\mathrm{H}$-bond connectivities in photochromic spiropyrans and their merocyanines sensed by ${ }^{13} \mathrm{C}$ deuterium isotope shifts," Physical Chemistry Chemical Physics, vol. 2, no. 1, pp. 53-56, 2000.

[42] G. Cottone, R. Noto, and G. La Manna, "Theoretical study of spiropyran-merocyanine thermal isomerization," Chemical Physics Letters, vol. 388, no. 1-3, pp. 218-222, 2004.

[43] E. Scrocco and J. Tomasi, "Electronic molecular structure, reactivity and intermolecular forces: an euristic interpretation by means of electrostatic molecular potentials," in Advances in Quantum Chemistry, P. Lowdin, Ed., pp. 115-193, Academic Press, New York, NY, USA, 1978. 

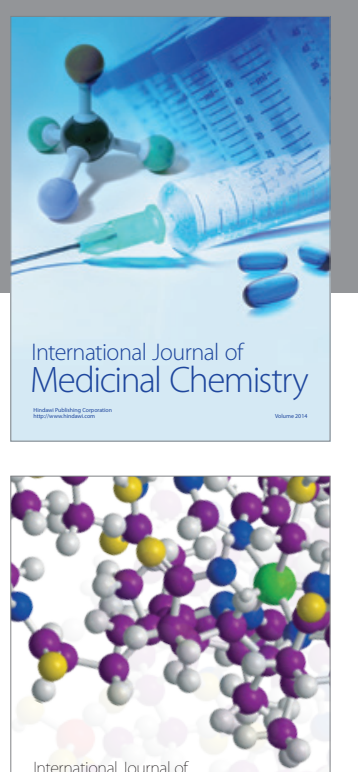

Carbohydrate Chemistry

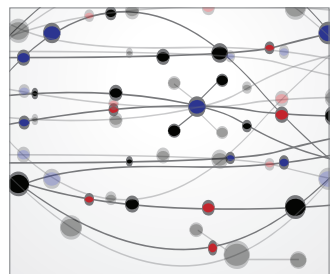

The Scientific World Journal
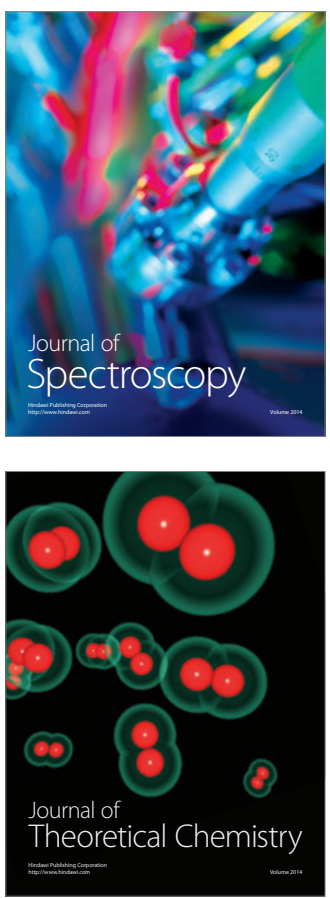
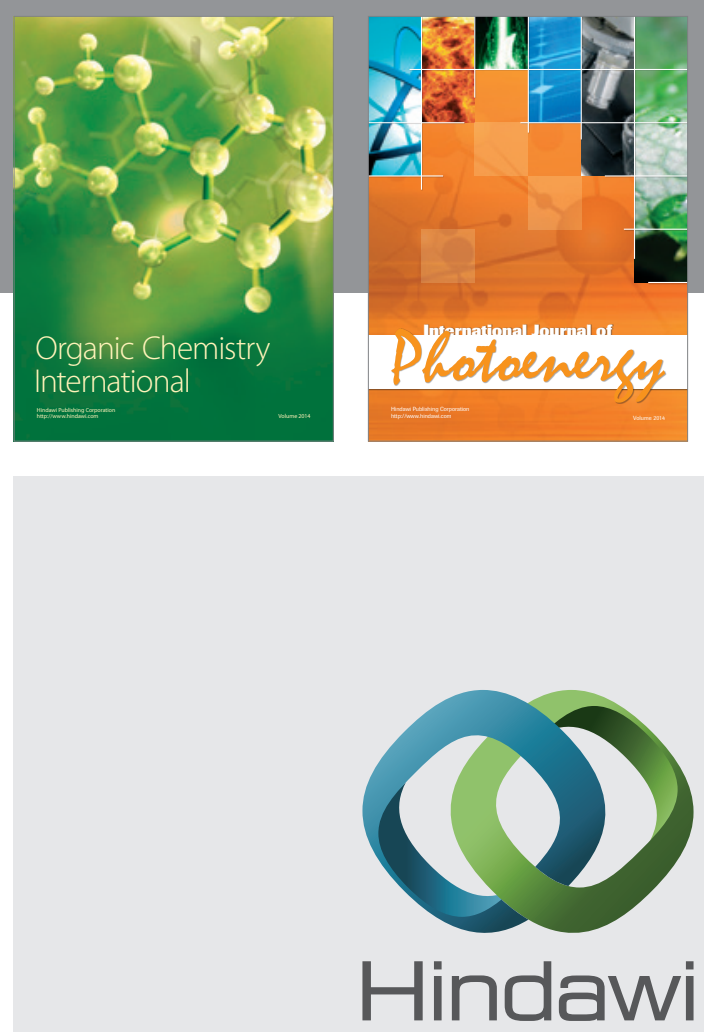

Submit your manuscripts at

http://www.hindawi.com

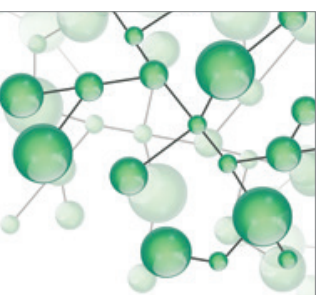

International Journal of

Inorganic Chemistry

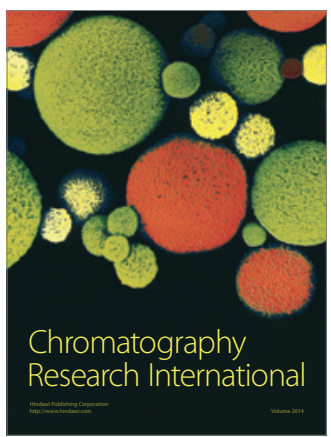

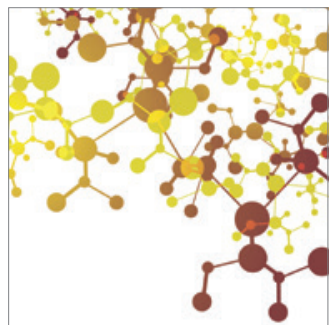

Applied Chemistry
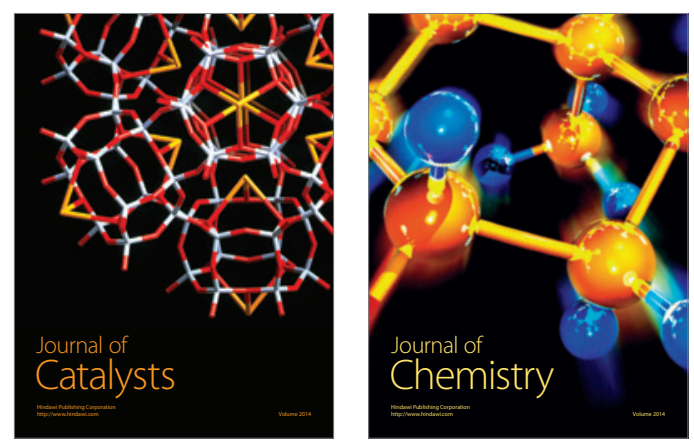
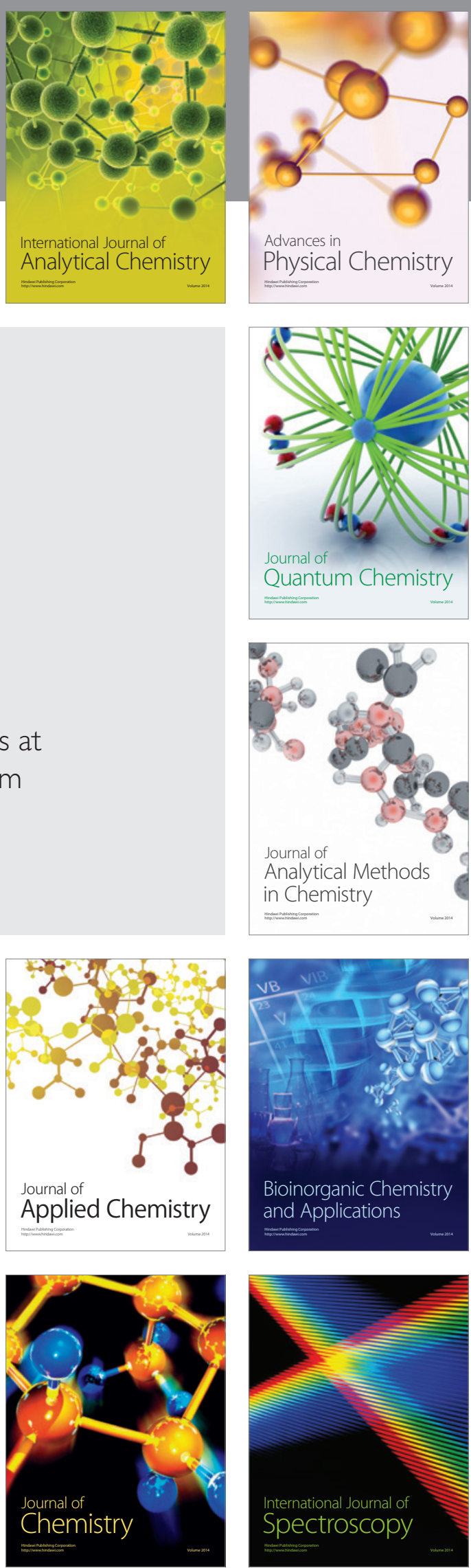